\title{
Ideas de los adolescentes mexicanos sobre la migración hacia los Estados Unidos. Elementos de reflexión para la praxis y política educativas
}

\author{
Mexican adolescents' ideas about migration towards \\ the United States as elements for reflection in praxis \\ and educational policies
}

Evelyn Diez-Martínez* ${ }^{*}$ Ricardo Naféz Arvizu**

\begin{abstract}
RESUMEN
El aumento considerable de la migración irregular de niños y adolescentes en México los expone a riesgos que atentan contra sus derechos humanos fundamentales. Son escasos los estudios en adolescentes sobre su comprensión acerca de este fenómeno social y del impacto del mismo en sus vidas y el futuro de las mismas. Se realizó una investigación con 69 alumnos de entre 13 y 17 años en una comunidad de alto índice de expulsión, aplicando un cuestionario que incluyó preguntas acerca de la situación de migración entre sus propios familiares, sus deseos de migrar, las razones y formas de realizar la migración, y temas sobre trabajo y migración. Los resultados muestran que los sujetos poseen diversas nociones sobre por qué y cómo se realiza la migración en sus comunidades y señalan claramente que muchos de ellos quisieran emigrar para lograr una movilidad social y una mejor forma de vida.
\end{abstract}

Palabras clave: concepciones sobre migración, adolescentes, educación

\section{ABSTRACT}

The considerable increase in irregular migration of children and adolescents in Mexico is exposing them to risks that threaten their fundamental human rights. In this regard, studies in adolescents about their understanding of the impact of the phenomenon of migration in their lives and their future have been scarce. A questionnaire was applied to 69 students between 13 and 17 years of age, sampled from an exclusion community. The questionnaire included questions concerning the situation of migration among their family members, their wish to become migrants, the reasons and forms to perform migration and some of their ideas about migration and labor. Results show that adolescents possess different notions about why and how migration is achieved in their communities and they also provide evidence that several adolescents questioned would like to emigrate, mainly to the United States as form of developing social mobility and a better way of life.

Key words: migration conceptions, adolescents, education

\footnotetext{
* Facultad de Psicología de la Universidad Autónoma de Querétaro; contacto: evelyn@uaq.mx

" Los autores agradecen el apoyo parcial del Consejo Nacional de Cienica y Tecnología (CONACyT) a este proyecto, mediante una beca otorgada a Ricardo Naféz para realizar sus estudios de maestría y el trabajo de campo de esta investigación.
} 


\section{Introducción}

Los autores del presente texto están interesados en el estudio de las formas en que se construye y adquiere a lo largo de la nińez, la adolescencia y la juventud, el conocimiento sobre la organización social y las instituciones que de esta se desprenden.

Los niños y los jóvenes devienen, poco a poco, en parte de las actividades de la organización estructural de su comunidad y en muchas ocasiones, en el caso de la migración, son ellos los principales beneficiados o los perjudicados de lo que brinda económicamente la partida de algún familiar como migrante al extranjero.

Hasta hace algunas décadas, la condición de migrante estaba reservada sobre todo para los adultos; sin embargo, en la actualidad, los niños y jóvenes no solo ven a los mayores migrar, sino que por un número de razones ellos mismos se ven obligados a hacerlo, a tal grado, que la migración de nińos y adolescentes acompańados o no acompañados plantea a estos una nueva perspectiva poco alentadora en el conocimiento de los fenómenos sociales de los que están rodeados, y en los que participan voluntaria o involuntariamente (ACNUR, 2013; Ceriani, 2013; CIDH, 2013; Van Dijk, 2005).

Los progresos que niños y adolescentes realizan en la comprensión de la organización social de sus comunidades y de los problemas de la misma, se ligan estrechamente al tipo y a la calidad de sus experiencias sociales, tanto como a sus estructuras intelectuales, que les permiten construir conceptualizaciones del mundo. Así, su conocimiento sobre lo social se lleva a cabo analizando experiencias, obteniendo información, y elaborando teorías acerca de lo experimentado (Enesco et al., 1995; Barret y Buchanan-Barrow, 2005). La comprensión de los niños, adolescentes y jóvenes sobre cómo está organizada su sociedad en términos económicos, políticos y sociales, y sobre cómo se conforman las instituciones, las reglas, y los valores que las generan y mantienen es una actividad continua y dinámica a lo largo de su desarrollo, y ha sido estudiada desde hace varias décadas por diversos autores desde la Psicología del Conocimiento Social (Furnham y Stacey, 1991; Barrett y Buchanan-Barrow, 2005; Enesco y cols., 1995; Delval, 2013; Diez-Martínez, 2012). 
En Querétaro, investigaciones relacionadas con la comprensión de la organización social y las aspiraciones ocupacionales en poblaciones de entre 12 y 18 años de diversos contextos socioeconómicos (Diez-Martínez, Ochoa, y Virues, 2008) han puesto al descubierto que, a pesar de los deseos de alcanzar estudios universitarios, los sujetos aportan, con frecuencia considerable, respuestas relacionadas con el hecho de emigrar a los Estados Unidos (EU) como una opción ocupacional, y que esta alternativa justifica el hecho de desertar de la escuela tempranamente o apenas terminados los estudios de secundaria. En otra aproximación similar en el municipio de Huimilpan, Querétaro, Vargas y Diez-Martínez (2008) analizan las ideas de niños y adolescentes sobre la migración como posibilidad de futuro ocupacional. Las autoras registraron que los sujetos reportan que migrar es una opción para los jóvenes varones solteros que no eran buenos estudiantes, aunque no siempre fueran los que contaban con menores recursos, demostrando así que no era la pobreza extrema la única condición para migrar y que los jóvenes que se iban lo hacían, principalmente, por imitar a alguien. Otro estudio específico sobre la comprensión de la migración en niños y adolescentes es el de Van Dijk (2005), quien estudió a 550 niños, niñas y adolescentes de 12 comunidades rurales campesinas con alta intensidad migratoria y altos índices de marginación y pobreza en el estado de Guanajuato. Como resultado, refiere que 50\% de los sujetos de su investigación eran muy conscientes de que querían continuar estudiando hasta un nivel universitario; sin embargo, más de $60 \%$ de ellos respondieron que también eran conscientes de que, debido a su precaria situación económica, no podrían continuar sus estudios más allá del nivel secundaria.

Estos estudios apuntan a una estrecha relación entre los diversos problemas dentro de los ámbitos educativos y las decisiones de los nińos y jóvenes de emigrar.

Con el fin de continuar generando información sobre estas ideas de los niños y adolescentes respecto al fenómeno de la migración y de aportar elementos que puedan ser considerados para futuras aproximaciones educativas al tema se llevó a cabo la presente investigación. 
Ahora bien, independientemente de las aproximaciones al problema de la migración desde la Psicología del Desarrollo Social y desde la Educación, el análisis de las razones del fenómeno tanto en adultos como en niños y jóvenes involucra diversas causas y problemáticas que, de manera muy breve, revisamos a continuación, para contextualizar la temática de nuestro trabajo y establecer una discusión sobre nuestros resultados.

El fenómeno de la migración genera, a nivel global, problemas sociales para los países de origen, tránsito y destino, y compromete las políticas públicas de inmigración, de derechos humanos, de educación y tipo de ciudadanía que se concibe para los inmigrantes en los Estados-Nación de recepción. En México y en América Latina, en general, estas cuestiones son controversiales y fundamentales para el momento actual. En 2014, 23000 niños y adolescentes mexicanos no acompańados (NNA) fueron detenidos en la frontera México-EU, y en su mayoría fueron deportados a su lugares de origen (CONAPO, 2014; Ceriani, 2013). Por otra parte, $80 \%$ de los niños, niñas y adolescentes migrantes de origen mexicano que fueron entrevistados por el Alto Comisionado de las Naciones Unidas para los Refugiados (ACNUR, 2013), señalaron desear la reunificación familiar, solventar los gastos de sus parientes directos o buscar oportunidades de desarrollo laboral, debido a que, en varios casos, los padres se van a trabajar al extranjero y, por su estado de irregularidad, no pueden entrar o salir libremente de su nuevo país de residencia, quedándose los hijos en esta brecha de separación social. También miles de niños y adolescentes migrantes de México y América Central deciden dejar sus hogares porque provienen de entidades aquejadas por la pobreza, la violencia social u organizada o la disfuncionalidad familiar (ACNUR, 2013; CIDH, 2013, 2015).

Las teorías estructurales de migración internacional que parten del trabajo de Wallerstein (1974, citado por Massey, Durand y Malone, 2009), intentan exponer que el fenómeno es explicado no tanto por las decisiones individuales o familiares, sino por las condiciones macroestructurales de los mercados globales. Por lo que la migración puede ser entendida con base en el crecimiento de las empresas transnacionales hacia sociedades de mercado, que dan origen a poblaciones móviles predispuestas a emigrar. 
Así, este fenómeno se manifiesta con base en los procesos de producción del modelo capitalista que, al apropiarse de los recursos naturales y materias primas de los países de sociedades con bajo desarrollo, van tejiendo una serie de factores que obligan a estas poblaciones a emigrar.

Como ejemplos, en México, se encuentran los procesos de privatización e industrialización del campo, que instrumentan una lógica que prescinde de los campesinos, cuando los procesos de producción de alimentos son mecanizados e industrializados. Esta industrialización se aprovecha de la mano de obra barata, individualiza los sistemas de pago a los trabajadores y, por lo tanto, socava y debilita las formas y relaciones de producción comunitarias y tradicionales.

Mac Donald y Mac Donald (1964, citados por Pedone, 2004) son los primeros en mencionar el concepto de "cadena migratoria”, como las formas y procesos por los cuales los futuros migrantes reciben información sobre ciertas oportunidades, tienen acceso a transportes y reciben apoyos para su instalación inicial y posibilidades de empleo, puesto que tienen relaciones sociales directas con emigrantes que los han precedido. Posteriormente, dicho concepto sería integrado al de red migratoria, ya que esta está compuesta de cadenas migratorias y va más allá. Pedone (ibid.) señala que estas redes vinculan de manera dinámica a las poblaciones de la sociedad de origen y a las de llegada, trascendiendo a los actores individuales.

Durand y Massey (2003) describen la teoría de las redes migratorias como conjuntos de lazos interpersonales que conectan a los migrantes con otros que los precedieron en las zonas de origen y destino mediante nexos de parentesco, amistad y paisanaje. Estas redes tienen amplia presencia en una serie de aspectos relacionados con el hecho de migrar, por ejemplo, otorgando apoyo emocional, reduciendo la incertidumbre o los factores estresores relacionados con el viaje, mientras que, al momento de la llegada, ayudan a acelerar la adaptación al país de acogida, disminuyendo efectos negativos. Estas teorías brindan dos perspectivas: las circunstancias económicas macroestructurales y la mirada del entorno comunitario y familiar, ambas como predeterminantes que pueden orillar a los niños y adolescentes a salir de sus comunidades. 
Massey, Durand y Malone (2009) sostienen que, actualmente, la teoría de la nueva economía de la migración de los trabajadores se ajusta más a la descripción de los hechos y eventos que suceden en los fenómenos migratorios y que, a diferencia de la teoría neoclásica de la migración, las posturas actuales sobre el fenómeno sostienen que las decisiones no se toman por individuos, sino por unidades de personas interrelacionadas, como los son los núcleos familiares o a veces las comunidades enteras, con el objetivo de trascender las condiciones de precariedad que originan los mercados del país de origen, cuando mantienen un debilitamiento del capital, crédito y seguros que podrían ofrecer a sus ciudadanos.

México ocupa el segundo lugar en emigración internacional, con 13.2 millones de mexicanos que residen en otros países; por otra parte, solo $30 \%$ de mexicanos tiene estatus de ciudadanía en EU y solo $16.6 \%$ de los migrantes mexicanos tiene nivel de preparatoria o profesional (CONAPO, 2014; OIM, 2013). Estas situaciones plantean retos en materia de educación y del tipo de ciudadanía y políticas públicas que se conciben en México y los EU para estos colectivos. Al momento actual, los niños y adolescentes pertenecientes a comunidades de alto índice de emigración en México, no cuentan con políticas públicas y educativas que les brinden un soporte frente al creciente fenómeno de la emigración (Giorguli y Leite, 2009); tampoco tienen suficiente información para tomar una decisión acertada respecto a sus aspiraciones ocupacionales (Diez-Martínez y Ochoa, 2014). Aunado a lo anterior, no cuentan con información respecto a los riesgos que implica la migración irregular, ni sobre los derechos humanos que los asisten, como alternativas para reflexionar sobre su migración.

Según datos del INEGI (2010), de los jóvenes o adolescentes que tienen entre 15 y 19 años, solo $57 \%$ asiste a la escuela, quedando $43 \%$ de esta población sin continuar sus estudios. En el estado de Querétaro, lugar donde se realizó esta investigación, el grado promedio de escolaridad de la población de 15 años o más, es de 8.9 grados, lo que equivaldría a prácticamente haber terminado la educación secundaria.

Muñiz (2001) sostiene que, en las zonas rurales de México, los niños en edades entre 7 y 11 ańos mantienen un alto porcentaje de asistencia (96\%); después de esa edad y hasta los 16 
años, los porcentajes disminuyen a menos de la mitad. También enfatiza que el rezago escolar de nińos y adolescentes es mucho mayor en zonas rurales con pobreza extrema, que en comunidades donde no hay pobreza.

Al respecto, Macías y Reyes (2004) realizaron un estudio con 779 trabajadores migrantes que residen en Nueva York, originarios de comunidades de expulsión de los estados de Puebla y Tlaxcala, buscando la relación entre la migración, la educación y el trabajo. Los autores encontraron que la razón más importante para abandonar la educación y tomar la decisión de migrar fue la poca capacidad de las localidades y comunidades de origen para generar empleo. También sostienen que los temas que se abordan en la educación formal de primaria, secundaria y preparatoria, poco tienen que ver con las labores que desempeñarán en sus futuros trabajos, y que esto acentúa el desinterés de los alumnos por continuar estudiando como una meta de vida. En este sentido, refieren que el contexto educativo que establecen las comunidades de expulsión está creando una especie de cultura que merma el proyecto de vida que podría basarse en la generación de una trayectoria educativa. Así también refieren que, en las comunidades rurales expulsoras de migrantes, los jóvenes reciben de forma constante información sobre experiencias asociadas con los éxitos en función de la movilidad social, el estatus y el poder que logran los familiares o amigos que se van a los EU, lo que robustece una cultura que beneficia la idea de migrar y actúa en detrimento de lo educativo.

Levine (2007), en un estudio sobre el estado actual de la escolaridad de migrantes mexicanos en EU, refiere que los latinos y los afroamericanos son las minorías étnicas y raciales que tienen menor nivel de escolaridad, puesto que mientras solo $12.4 \%$ de los inmigrantes asiáticos no tienen el certificado de High school (bachillerato), $49 \%$ de migrantes mexicanos no lo posee. Por otro lado, mientras que solo $7.8 \%$ de los inmigrantes mexicanos tiene un título universitario, $49 \%$ de los asiáticos lo posee. Cuando los nińos y adolescentes migrantes mexicanos se van a vivir a los EU, también están expuestos a lo que Levine (2007) describe como discriminación escolar, que consiste en la estratificación y segregación racial de estos en las escuelas oficiales, por medio de la asignación de menos recursos e infraestructura para las escuelas 
que están insertas en los barrios donde viven, además de incurrir en prácticas deficientes por parte de los docentes, todo lo que tiene como consecuencia que las aspiraciones y expectativas de los niños y adolescentes migrantes mexicanos se vayan truncando.

Dreby y Stutz (2012) investigaron a 1273 estudiantes de la región mixteca del estado de Oaxaca, buscando encontrar la relación entre las experiencias de los niños ante la migración de sus familiares y sus aspiraciones educacionales; ellos encontraron que la migración de la madre afecta de diversas maneras su desempeño escolar. Por otra parte, cuando solamente el padre emigra, no hay una afectación significativa en su desempeño educativo. Asimismo, realizaron 51 entrevistas a niños y niñas cuyas madres no habían migrado, encontrando que mantenían mayores motivaciones de permanecer en la escuela. A su vez, cuando ambos padres habían emigrado, las condiciones emocionales de los niños y niñas fungían como un factor decisivo en la disminución de sus aspiraciones educacionales. Los mismos autores refieren que, si bien una de las razones por las que los padres migran es porque desean brindarles mejores oportunidades laborales y educativas a sus hijos, sin advertirlo, muchas veces sus hijos tenderán a seguir su ejemplo como futuros migrantes, confirmando con esto la predominante cultura de la migración y la conformación de actitudes pro migratorias en los niños y adolescentes. Los mismos autores refieren que existen relaciones entre la motivación de logro y las iniciativas de ayudar a sus padres en los niños y adolescentes. Así, el hecho de que los padres migren, con los sacrificios que esto implica, puede motivar a los niños y adolescentes (a través de la culpa que pueden sentir), a que terminen adoptando un patrón que los autores llaman "logro compensatorio", que los llevaría a emigrar. Agregan, igualmente, que los niños y adolescentes también presentan variadas afectaciones emocionales como resentimientos y dificultades para relacionarse con sus padres, una vez que estos han regresado de EU.

McKenzie y Rapoport (2006) enfatizan que, en México, los posibles efectos de la migración internacional apuntan a que los proyectos educativos son sustituidos por la emigración como medio idóneo de movilidad social, lo que afecta la asistencia escolar de niños entre 12 y 15 años de edad y de niñas entre 16 y 18 .

Diez-Martínez y Ochoa (2014) realizaron un estudio con 470 adolescentes mexicanos entre 12 y 18 años -de los cuales una 
tercera parte provenía de una comunidad de expulsión-, para conocer cuáles eran sus aspiraciones ocupacionales en primera y segunda opción; se encontró que existía, en todos los sujetos, una motivación para cursar estudios universitarios, aunque en un alto porcentaje aspiraban a ingresar a carreras tradicionales como derecho, ingeniería o medicina, cuya demanda es mayor que la capacidad de admisión de las universidades, lo que produce una falta de congruencia entre lo que se quiere estudiar y la oferta laboral que existe en el país, y genera un círculo de pobreza ante la imposibilidad de seguir estudiando, la falta de empleos y las posibles aspiraciones ocupacionales como migrantes.

Otro de sus resultados es el hecho de que los sujetos, desde los diez años, comienzan a discriminar acerca de qué ocupaciones no elegirían desempeñar en un futuro, con base en sus logros o fracasos académicos en determinadas materias. Las autoras advierten que, actualmente, no existe un adecuado abordaje de la orientación y la formación vocacional en los grados de sexto de primaria y secundaria, y que la orientación que se brinda en el nivel de bachillerato presenta deficiencias. Estos hallazgos muestran la necesidad apremiante de promover más investigación y programas de intervención respecto a la orientación vocacional de los niños y adolescentes de México, ya que con base en esta deficiente instrucción, niños y adolescentes toman decisiones tan trascendentales como elegir una ocupación, carrera u optar por la migración.

Es interesante el hecho de que, al analizar la relación del currículo nacional con el tema de la migración en México, se encuentra que la Secretaría de Educación Pública (SEP) propone la utilización de marcos curriculares para poder flexibilizar el currículo de la educación básica, siempre en consonancia con el Plan Nacional y los programas de estudio (SEP, 2011; SEP,2013); sin embargo, se encuentra que dicha orientación pedagógica está diseñada solo para una línea de acción, que es la migración interna de los niños y adolescentes hijos de padres jornaleros, el menor migrante repatriado en México (Programa Binacional de Educación Migrante (PROBEM), y el Proyecto Educación Básica sin Fronteras (Atención educativa intercultural a estudiantes de educación básica inmigrantes y emigrantes), pero no se ha orientado a instrumentar sus programas y planes de estudio en un aspecto muy importante, que es el de propiciar la atención y la reflexión 
sobre la emigración irregular de niños y adolescentes mexicanos para mitigar los efectos negativos de su salida del país.

En la multicausalidad de la migración de los niños, adolescentes y jóvenes en nuestro país, parece claramente manifiesta una relación entre la falta de estudios, de perspectivas y aspiraciones ocupacionales entre estos y sus decisiones de emigrar en busca de un futuro más promisorio.

El trabajo que describimos a continuación, se llevó a cabo con la idea de analizar cierto tipo de conocimiento en los adolescentes, de generar datos fundamentados en la investigación para diseñar programas educativos que los retomen y proporcionen información y orientación a adolescentes acerca de la migración.

\section{Metodología}

Se aplicó un cuestionario de elaboración propia, considerando información recabada por estudios anteriores, con 36 preguntas abiertas y una de opción múltiple, a 69 adolescentes que asistían a escuelas públicas en el municipio de Huimilpan, Querétaro, en México. Los sujetos fueron todos los adolescentes que asistían de forma voluntaria a un taller informativo acerca de varias temáticas de interés para adolescentes, organizado por el Sistema Nacional para el Desarrollo Integral de la Familia (DIF), dentro del cual uno de los autores fungía como participante de apoyo. La aplicación del cuestionario tuvo una duración de 30 minutos y se realizó en una sesión.

Las edades de los sujetos fluctuaban entre los 13 y 17 años, con escolaridad de primaria y secundaria. Se trabajó con $43 \mathrm{mu}-$ jeres y 26 hombres. Como objetivo específico, se buscó conocer las ideas de los adolescentes acerca de la migración en general y en su comunidad, los riesgos de la migración, sus aspiraciones ocupacionales y educativas, y sus apreciaciones sobre su comunidad.

\section{Resultados}

Con las respuestas de los sujetos se realizó un análisis inicial que permitió agruparlas en tipos similares, en el sentido de una lógica o argumentación semejante. Estos tipos de respuesta son presen- 
tados en porcentajes de frecuencia de aparición en cuadros elaborados por los autores. A continuación presentamos algunos de los resultados que consideramos interesantes en términos de nuestro objetivo general.

CUADR0 1. ¿Alguna vez has pensado irte a los EU?

\begin{tabular}{|l|c|}
\hline Sí, para ganar dinero, mejor vida o tener trabajo & 33 \\
\hline Sí, por causas familiares o de reunificación familiar & 16 \\
\hline Sí, para conocer o viajar & 13 \\
\hline Sí, para Estudiar & 9 \\
\hline No & 28 \\
\hline
\end{tabular}

En el cuadro 1 se observa que $71 \%$, prácticamente la totalidad de los sujetos, ha contemplado la idea de emigrar hacia EU, lo que confirma la existencia de una cultura de la migración, ya anticipada por varios autores (Durand y Massey, 2003; Massey, Pren y Durand, 2009). Las principales razones por las que lo harían son para ganar dinero, tener una mejor vida o trabajo; en segundo lugar, se encuentra que varios de los adolescentes entrevistados tienen familiares que ya residen en EU y anhelan la reunificación familiar; en tercer lugar, se encuentra la motivación de viajar y conocer nuevos lugares y, por último, les gustaría ir a los EU para estudiar.

CUADRO 2. En tu familia, ¿te han dicho que deberías irte a EU?

\begin{tabular}{|l|l|}
\hline N0 & 70 \\
\hline Sí & 23 \\
\hline
\end{tabular}

En el cuadro 2 se observa que, a pesar de que $70 \%$ de los entrevistados refiere que sus figuras parentales no les han sugerido directamente que deberían emigrar hacia EU, al menos $23 \%$ de los mismos sí ha escuchado de sus padres deseos o ideas relacionadas a que deberían viajar a ese país. Como se verá más adelante, uno de los principales factores por los que los adolescentes deciden migrar está relacionado con la búsqueda y reintegración con sus familiares que residen en EU, lo que confirma la afinidad con las teorías sobre las redes migratorias, que sostienen varios autores (Durand y Massey, 2003; Pedone, 2004; Portes, 2007). 
CUADRO 3. En tu familia cercana, ¿hay jóvenes que ya se han ido a EU?

\begin{tabular}{|l|c|}
\hline Sí & 96 \\
\hline NO & 4 \\
\hline
\end{tabular}

En el cuadro 3 se observa que $96 \%$ de los adolescentes entrevistados manifiesta que, efectivamente, tienen familiares que se encuentran en EU, principalmente primos, tíos, hermanos, padre y madre. Esto confirma la relación entre los conceptos de redes migratorias y cultura de la migración de Durand y Massey (2003), Pedone (2004) y Portes (2007), quienes postulan que la migración México-EU se sostiene y continua acrecentándose, gracias a las cadenas y redes sociales que se tejen en uno y otro país, ya que los migrantes de segunda o tercera generación tienen más facilidades para realizar su viaje cuando tienen familiares y amigos que les ayudan con contactos, espacios laborales y recursos económicos para tener éxito en su objetivo de establecerse en el país del norte. Asimismo, sostienen que las redes migratorias son uno de los factores que gestan la conformación de las comunidades transnacionales, entre las que se da un amplio tránsito de bienes económicos y sociales en espacios establecidos tanto en México como en EU. A esto se debe que la comunidad de Huimilpan, a la que pertenecen los sujetos de la muestra, mantenga una constante tendencia a favorecer y perpetuar la cultura de la migración, con base en el alto índice de redes de familiares y amigos que ya viven en el vecino país.

CUADRO 4. Elige el orden de importancia de las razones por las que te irías a EU

\begin{tabular}{|l|l|}
\hline Para tener un mejor trabajo & 32 \\
\hline Para ganar dinero & 28 \\
\hline Para viajar y conocer nuevos lugares & 22 \\
\hline Para vivir con mi familia & 18 \\
\hline
\end{tabular}

En el cuadro 4 se muestra que las principales razones por las que los adolescentes eligen arriesgar sus vidas para emigrar a EU son económicas (60\%, sumando las dos primeras respuestas); en tercer lugar, que lo harían motivados por las ambiciones de conocer y viajar a un nuevo lugar $y$, por último, que lo harían por promover una reunificación familiar. Esto constata, por una parte, que las 
necesidades económicas que perciben en su entorno son uno de los múltiples factores para tomar la decisión de emigrar a otro país y, por otra, el hecho de que los sujetos quisieran irse a EU como forma de conocer nuevos lugares viajando. Domínguez y Polo (2006), en un estudio sobre las motivaciones para emigrar de 580 adolescentes mexicanos, encontraron que la dimensión del autodesarrollo es, en ocasiones, incluso más importante que las razones económicas y hace hincapié en la búsqueda de situaciones nuevas y diferentes que caracterizan a la población adolescente.

Estos resultados muestran que las motivaciones de los adolescentes para emigrar son heterogéneas y podrían variar en las distintas regiones de México, aunque no se encontraron estudios que hayan analizado semejanzas o diferencias en los deseos y motivaciones de los jóvenes para emigrar por regiones específicas.

CUADR0 5. En tu familia, ¿qué profesionistas existen o qué oficios desempeñan?

\begin{tabular}{|l|c|}
\hline Albañil & 40 \\
\hline Licenciatura & 21 \\
\hline Jardinero & 13 \\
\hline Agricultor o Ganadero & 11 \\
\hline Ama de casa & 11 \\
\hline No respondió & 4 \\
\hline
\end{tabular}

Con respecto a las profesiones y oficios que los adolescentes observan dentro de su núcleo familiar, $74 \%$ de los entrevistados refiere que sus familiares cercanos desempeñan algún oficio, mientras que solo 26\% afirma que ejercen alguna profesión. El oficio más frecuente es el de albañilería o construcción, con $36 \%$; en segundo lugar refieren varias licenciaturas y, en tercero, la jardinería. Estos datos muestran una fuerte tendencia entre los adultos de la comunidad a desempeñar ocupaciones que no requieren estudios o calificaciones institucionales, y que proporcionan ejemplos para las aspiraciones ocupacionales de los adolescentes que, al no contar con orientación vocacional desde el fin de la educación primaria, optan por continuar con los oficios ejercidos por sus padres, o por escuchar que son estos los requeridos en los EU. Diez-Martínez y Ochoa (2014) refieren que, en las comunidades de México, los niños y adolescentes, por lo general, siguen 
el ejemplo de sus progenitores en sus futuras ocupaciones, ya que el conocimiento sobre el trabajo y la identidad ocupacional se desarrolla en la medida en que los niños y jóvenes participan en las prácticas comunitarias, donde los oficios son prácticamente una herencia social, que puede o no favorecer la movilidad social.

Kandel y Kao (2001), a su vez, señalan que, gracias a las redes sociales migratorias de amigos o familiares que ya están establecidos, el proyecto de convertirse en migrante se vuelve más atractivo que invertir en un proyecto que implique continuar la educación más allá de la secundaria.

CUADRO 6. ¿Cuánto dinero se necesita para hacer el viaje a EU?

\begin{tabular}{|l|l|}
\hline No sabe & 40 \\
\hline Más de 10000 Pesos & 35 \\
\hline Menos de 10000 Pesos & 25 \\
\hline
\end{tabular}

Con respecto al monto necesario para emprender el viaje, se observa que $40 \%$ de los entrevistados manifiesta no saber o no tener idea aproximada de alguna cantidad para sufragar los gastos para migrar EU. Estos datos ponen en evidencia que los adolescentes no tienen conciencia de índole económica lo que, aunado la falta de una orientación vocacional previa y a la de un plan de vida, facilita que busquen emigrar con una mínima planeación, de manera casi impulsiva y con mayores probabilidades de fracasar. Cabe aclarar que, frente a la multiplicidad de respuestas, se estableció un valor aproximado con base en lo que especificaran los adolescentes, por lo que solo $35 \%$ de la población respondió más de diez mil pesos, una cifra relativamente coherente frente a las circunstancias y costos que implica hacer un viaje de tal magnitud. Por último, 25\% de los entrevistados manifestó que se requería menos de esa cantidad para realizar dicho viaje.

$\mathrm{Al}$ respecto, se ha señalado antes la complejidad de nuestras problemáticas educativas en Diez-Martínez (2015), cuyo analisis sobre los niveles de alfabetización económica de los adolescentes mexicanos muestra que esta habilidad es practicamente inexistente en el currículo nacional, y que tanto los estudiantes de estas clases sociales como de niveles socioeconómicos medios y altos tienen ideas muy incipientes sobre situaciones e intercambios 
económicos sencillos, lo que deja una brecha más que salvar en las competencias requeridas para la vida cotidiana de los jóvenes de nuestro país.

CUADRO 7. ¿Escribe una razón por la que te gusta vivir en tu comunidad?

\begin{tabular}{|l|c|}
\hline Porque es tranquilo, hay naturaleza, el campo y está limpio & 46 \\
\hline Porque están mi familia y amigos & 22 \\
\hline Porque me gusta, simplemente & 21 \\
\hline Porque hay libertad & 11 \\
\hline
\end{tabular}

Se exploraron las razones por las que los adolescentes gustan de vivir en su comunidad, pues se consideró que sus respuestas serían valiosas en torno a los temas que pueden influir en la toma de decisiones antes de emigrar. De esta forma, se observa que $46 \%$ de los entrevistados está de acuerdo en que la principal razón por las que aprecian su comunidad es por los recursos naturales que conservan, por la tranquilidad que experimentan y por la calidad de vida de la que gozan por vivir en un lugar sin contaminación ambiental. En este sentido, resulta importante reflexionar acerca de la importancia que podría tener educar a los adolescentes en el desarrollo sustentable, ya que es un tema que les genera interés y curiosidad, toda vez que son conscientes de las riquezas naturales del entorno en el que viven. Al respecto, Toledo y Ortiz-Espejel (2014) sostienen la importancia de documentar e instituir proyectos de intervención comunitaria en México, con el objetivo de promover un desarrollo local sustentable en este tipo de comunidades, ya que contribuirían a revertir las altas tasas de emigración generando fuentes de trabajo.

CUADRO 8. ¿Escribe una razón por la que no te gusta vivir en tu comunidad?

\begin{tabular}{|l|c|}
\hline $\begin{array}{l}\text { Porque no hay trabajo; no hay recursos; no hay desarrollo; } \\
\text { no hay oportunidades ni apoyo del Estado. }\end{array}$ & 69 \\
\hline Por problemas de relaciones entre los miembros de la comunidad & 17 \\
\hline No hay posibilidades de estudiar & 7 \\
\hline No hay razón & 5 \\
\hline No sé & 2 \\
\hline
\end{tabular}


Resulta significativo, en el cuadro 8, que 69\% de los informantes respondió que no gustaba de vivir en su comunidad debido a la falta de trabajo y de oportunidades de desarrollo personal y laboral, así como a que no encontraba apoyo por parte del Estado. Estos datos confirman los seńalamientos de autores como Macías y Reyes (2004) quienes, analizando las respuestas a estos temas en sujetos de edades similares, han puesto nuevamente en evidencia que una de las razones mas importantes de la migración es el hecho de que las comunidades de origen no generan empleos suficientes para promover que los jóvenes permanezcan en estas. Por otra parte, en este estudio, 17\% atribuyó su falta de disposición a problemas con miembros de la comunidad y únicamente 7\% manifestó que es debido a la falta de oportunidades para continuar su educación. En este sentido, es posible que los entrevistados no estén continuando sus estudios, no tanto por las posibilidades económicas o materiales para continuar hacia el nivel medio superior, sino por el hecho de que continuar estudiando no es algo que esté dentro de sus aspiraciones ocupacionales; por el contrario, los adolescentes mantienen actitudes pro migratorias, como lo han señalado anteriormente varios autores (Dreby y Stutz, 2012; Kandel y Kao, 2001).

Relacionando esto con los resultados del cuadro 5, donde se muestra que solo $26 \%$ de los familiares de los adolescentes con estudios se dedica a alguna profesión, podríamos inferir que el bajo desarrollo económico, humano, más la cultura de la migración y la influencia de sus familiares, son factores que influyen en los jóvenes para elegir la migración como una ocupación y perspectiva de movilidad social.

CUADR0 9. ¿Cómo te imaginas que vas a cruzar la frontera?

\begin{tabular}{|l|c|}
\hline Irregularmente & 44 \\
\hline Regularmente, en avión, camión, con visa, papeles & 38 \\
\hline No sé. No me imagino & 14 \\
\hline En compañ́a de otra persona & 4 \\
\hline
\end{tabular}

Destaca, en el cuadro 9, que casi la mitad de los adolescentes entrevistados (44\%), en caso de optar por convertirse en migrantes, buscarían cruzar la frontera de México a EU de manera 
irregular, mientras que $38 \%$ expresa el deseo de hacerlo por vías regulares y con la presencia de documentos, lo que implica que, siendo conscientes de los riesgos, desearían poder ejercer su derecho a migrar bajo las condiciones más seguras posibles. También se encuentra que hay ambigüedad entre legalidad e ilegalidad, cuando mencionan que cruzarían en auto o en camión, ya que no especifican bajo qué modalidad lo harían. Es importante aclarar que el uso de los términos legal o ilegal plantea una visión de la migración como un acto punible y perseguible, cuando, en décadas recientes, numerosas organizaciones han propuesto usar los términos regular e irregular para descriminalizar un derecho humano como la migración, ya que ningún ser humano puede ser "ilegal". Ante esto, diversos autores e instituciones usan los términos regular/irregular, para describir la situación legal de los NNA migrantes (Ceriani, 2013; CIDH, 2013, 2015; IDC, 2013).

CUADRO 10. ¿Qué obstáculos te imaginas que podrías tener en tu viaje hasta llegar a EU?

\begin{tabular}{|l|l|}
\hline Ser capturados por alguna autoridad, migración o policías & 29 \\
\hline Obstáculos que ponen en peligro la vida & 26 \\
\hline Otros peligros & 28 \\
\hline Problema del idioma & 6 \\
\hline Ninguno & 4 \\
\hline No sé & 6 \\
\hline
\end{tabular}

Es notable que, en el imaginario que los adolescentes respecto al tema de los riesgos u obstáculos que podrían tener en su proceso migratorio, se encuentra que $29 \%$ sostiene que podría ser capturado por alguna autoridad, ya fuera perteneciente al Instituto Nacional de Migración (INM), la policía federal o el ejército.

Con respecto a los alumnos que contestaron dentro de la categoría "Ningún peligro", algunos de ellos afirmaron que no tendrían obstáculos debido a que realizarían su viaje de manera regular, con presencia de sus documentos legales.

En cuanto a la categoría "Problema del idioma", solo $6 \%$ manifiesta, explícitamente, esta dificultad; esto nos permite deducir que la mayoría de los adolescentes entrevistados no está plenamente consciente de que este podría ser uno de los mayores obstáculos que tendrían al llegar a EU. 


\section{CUADR0 11. ¿Crees que existen riesgos? ¿Cuáles?}

\begin{tabular}{|l|c|}
\hline Riesgos de Muerte & 33 \\
\hline Ahogamiento & 19 \\
\hline Deshidratación & 23 \\
\hline Detención por migración & 12 \\
\hline Asalto o secuestro por grupos delictivos & 8 \\
\hline No sabe o no especifica & 5 \\
\hline
\end{tabular}

En cuanto a los peligros de migrar, es posible observar que $38 \%$ de los entrevistados manifiesta que los riesgos asociados a hacerlo de manera irregular están directamente vinculados con la posibilidad de morir en el intento y, si se suman los porcentajes de las categorías "Deshidratación" y "Ahogamiento", tendríamos $83 \%$ de adolescentes que afirman conocer que los riesgos de la migración son altos y pueden llevar a alguna forma de muerte.

CUADRO 12. Supongamos que hay niños y adolescentes que no logran cruzar la frontera, ¿qué te imaginas que les pasa?

\begin{tabular}{|l|c|}
\hline Muerte por diversas causas & 41 \\
\hline Detención o cárcel & 30 \\
\hline Deportados o regresan con su familia & 20 \\
\hline Condiciones que no ponen en riesgo su vida & 9 \\
\hline
\end{tabular}

Respecto a la cuestión de las consecuencias que podrían tener los adolescentes migrantes que no logran cruzar la frontera, en el cuadro 12 es posible observar que los entrevistados respondieron, en $41 \%$, que imaginan que morirían por diversas causas.

En segundo lugar, el 30\% respondió que los adolescentes serían detenidos por alguna figura de autoridad institucional, como podría ser el INM o el DIF, con la consecuencia de que fueran encarcelados o privados de sus derechos fundamentales por el solo hecho de ser menores de edad migrantes. Es llamativo que muchos adolescentes que respondieron esta cuestión, también relacionaban la idea de detención y la idea de fallecer como principales elementos negativos en sus respuestas. En tercer lugar, 20\% respondió que los adolescentes eran deportados o retornados con sus familias. Por último, solo 9\% proporcionó respuestas de lo que imaginan podría suceder a los niños y adolescentes migran- 
tes, cuyo principal contenido fue de orden emocional ligado a la tristeza, la soledad o el abandono.

\section{Conclusiones}

Los resultados del cuestionario muestran algunas de las conceptualizaciones de niños y adolescentes sobre ciertos tópicos relacionados con la migración. También cómo, desde la niñez y la adolescencia, se construyen las bases para las conductas adultas y grupales que componen los cimientos de la cultura de la migración y las redes migratorias. De acuerdo a las teorías psicológicas del desarrollo del conocimiento social, el nivel de comprensión, estructuración y utilización de este por el sujeto cognoscente, está mediado por los siguientes factores; el contexto psicológico de las interacciones sociales individuales y colectivas, las condiciones socioeconómicas de referencia y la transmisión social determinada por la educación y la cultura.

Los datos arrojados por nuestra muestra de adolescentes, parecen evidenciar un conocimiento bastante semejante en todos los sujetos con respecto a la migración, que de alguna manera es un punto de discusión y de marcaje social dentro de la comunidad que los sujetos perciben desde edades tempranas. Sin embargo, consideramos que análisis realizado en esta investigación, debería realizarse en sujetos de diversas edades, aun en adultos y contextos socieconómicos de referencia diferentes, para establecer el papel de cada uno de estos factores en el desarrollo del conocimiento social.

En cuanto los aspectos educativos que debían ser el fin último de investigaciones como la presente, creemos que se requiere una discusión interdisciplinaria para el abordaje de problemáticas tan graves en nuestro país, como la emigración de nuestros niños y jóvenes.

Giorguli y Leite (2009) sostienen que los adolescentes en México pueden ver interrumpida su educación debido al efecto de la cultura de la migración, donde al estar expuestos a observar el fenómeno de la migración como elemento de superación personal, pueden elegir hacerlo como una aspiración ocupacional. Igualmente, es de notar que, a pesar de tener nociones sobre las vicisitudes y peligros que conlleva la migración, la mayoría de los sujetos 
de la muestra ha pensado en intentar una posible migración a EU. Autores como Van Dijk (2005) y Giorguli y Leite, (2009) mencionan que los estados de la República mexicana que presentan un porcentaje de mayor deserción escolar, están relacionados con la existencia de una mayor intensidad migratoria en sus comunidades. Aunque, por otro lado, las remesas que reciben las familias pueden contribuir a retrasar la deserción escolar de los adolescentes, especialmente en hogares que dependen exclusivamente de ellas, no son un factor decisivo, pues tienen una diferente utilización en las distintas regiones y comunidades de emigrantes en México (Durand y Massey, 2003; Massey et al., 2009).

Varios autores (Rojas, 2005; Giorguli y Leite, 2009; Arnaut y Giorguli, 2010) coinciden que hay una carencia de políticas públicas que atiendan adecuadamente el problema de la migración y la educación en México. La SEP debería considerar la creación de un programa educativo específico para comunidades con alto índice de migración, adecuando el currículo con base en las necesidades de estas regiones, incluyendo los temas sobre la migración y aspiraciones vocacionales desde grados anteriores a la secundaria.

Se concluye que es deseable expandir las posibilidades de la educación formal como una herramienta necesaria para la educación sobre la migración, tanto en el diseño y planeación del currículo, como el del material didáctico.

Se puede pensar que un factor importante, pero no definitivo para lograr una educación incluyente y significativa en entornos comunitarios, precisa de la presencia de actores originarios de la misma población, capacitados en torno a las herramientas que brinda la mediación cognitiva y pedagógica. La impartición de talleres que busquen una reflexión acerca de la migración en comunidades de expulsión, podría tener un mejor impacto cuando se realice por parte de un mediador o profesor que sea originario de su propia comunidad, previamente capacitado tanto en información como en recursos pedagógicos. Esto facilitaría la impartición de este tipo de talleres, como ha sido señalado anteriormente (Baronnet, Mora, y Stahler-Sholk, coord., 2011), y sin buscar imponer ideas erradas, que se salgan o violenten el contexto cultural en el cual se han formado los niños y adolescentes originarios de estas comunidades. 
El programa del DIF, Estrategia de Prevención a Niñas, Niños y Adolescentes Migrantes y Repatriados No acompañados ( , 2012) no reconoce la migración como un derecho humano, y queda con labilidad frente a qué estrategias brindar a los jóvenes para que no emigren, ya que encontramos en varios estudios de comunidades con alto índice de expulsión (incluyendo esta investigación), causas macroeconómicas, costumbres y tradiciones, ritos de paso a la adultez que potencian y reproducen la cultura de la migración. El programa también podría adecuarse para que esté enfocado a que sus proyectos de contención de niños y adolescentes en riesgo de migrar irregularmente, den prevalencia al financiamiento no solo de fuentes de autoempleo, sino de becas y oportunidades para que estos adolescentes prosigan su educación. En este sentido, los talleres de migración que se imparten desde la plataforma oficial del SNDIF deberían considerar el derecho que tiene todo ser humano a migrar por diversas causas, entre ellas la reunificación familiar. La literatura al respecto refiere que la separación familiar por causas de migración de uno o dos de los padres, causa sufrimiento en los hijos que residen en su país de origen y disminuye su desempeño escolar y las aspiraciones ocupacionales (Castañeda y Buck, 2011; Donato y Duncan, 2011; Lu, 2014).

La prevención de la migración, a su vez, debería de estar enfocada a los riesgos de la que es irregular, cuando los niños y adolescentes viajan por la vía del tren o en camión, pero también se debe trabajar el derecho a migrar por medios que reduzcan al mínimo los riesgos, como en el caso de la migración como estudiante, la migración documentada y el asilo político.

Como ejemplo, Questa y Utrilla (2006) encontraron que, en las comunidades del Estado de México, la migración estaba disminuyendo gracias, en parte, a mayores posibilidades en la educación escolar, que permite a sus habitantes quedarse en su comunidad hasta terminar la secundaria o el bachillerato técnico en centros urbanos de menor escala, como Atlacomulco y Jilotepec.

Entre algunos de los argumentos expuestos por Giorguli y sus colaboradores (2012), respecto a las mejoras y sugerencias a los programas educativos en las comunidades de expulsión, encontramos la necesidad de que, en primera instancia, se fortalezca la infraestructura física de estas escuelas comunitarias, buscando 
mejorar la calidad y distribución de los materiales de aprendizaje, así como incrementar la presencia de equipos de cómputo e instalaciones adecuadas. En segundo lugar, incrementar el tiempo que los profesores y estudiantes pasan en el aula puede ser significativo para el involucramiento entre unos y otros, lo que facilita el logro de mayores aprendizajes. En tercero, realizar una adecuada vinculación de los planes de estudios con las oportunidades laborales de la zona geográfica de la comunidad de expulsión puede ocasionar que los estudiantes, paulatinamente, estén más inclinados a ver la escuela como un medio de concretar aspiraciones y oportunidades ocupacionales; este proceso debería ir acompañado de un adecuado asesoramiento en orientación vocacional a cada uno de los implicados.

En cuarto lugar, la capacitación a los maestros debe buscar que estos puedan asociar los temas de los planes de estudio con las vivencias y experiencias de migración que los adolescentes y sus familias han experimentado. Por último, el incremento de los financiamientos que diversos sectores puedan hacer para la coordinación de la investigación, educación y el desarrollo local pueden coadyuvar en detonar alternativas que contribuyan a revertir la migración.

Con base en lo señalado por Portes (2007), fomentar en los adolescentes la educación media superior y superior aumenta en 172\% y $200 \%$, respectivamente, las probabilidades de que estos migrantes se conviertan en ciudadanos activos que luchen por sus derechos en su país y en el país de destino, desde una perspectiva que busque una política transnacional. En cuanto mayor escolaridad tengan los migrantes, mayores comodidades y seguridades pueden tener en el país al que arriben, fortaleciendo también la probabilidad de pertenecer a organizaciones de migrantes que les ayuden a defender y a tener mayor acceso a sus derechos fundamentales y al estatus de ciudadanía; por otra parte, contribuyen a crear estereotipos positivos constituyéndose como "minorías modelo", como es el caso de la mayoría de los asiáticos que emigran a EU.

Se debería incluir en las escuelas secundarias comunitarias (especialmente en las que se conoce que mantienen alto índice de migración) en las materias de educación cívica (SEP, 2011), el tema de la migración con un desarrollo mayor, la educación formal puede potenciar un poco más el logro de aprendizajes 
significativos para los adolescentes que habrán de emigrar, brindándoles mayores competencias para enfrentar la vivencia, la adaptación, el manejo del idioma, aspirando a que cuenten con más recursos emocionales y de conocimiento que contribuyan a no presentar una experiencia traumatizante o de duelo continuo. Por otra parte, una adecuada orientación vocacional a lo largo de la formación secundaria puede potenciar que los estudiantes contemplen la posibilidad de ver como aspiración ocupacional continuar estudiando hasta el grado profesional, evitando tener que emigrar en condiciones peligrosas para su integridad.

Cárdenas y González (2013) apuntan a la necesidad de diseñar y proponer un taller a los maestros de secundaria para que tengan mayores herramientas para transmitir estos conocimientos a sus alumnos. Brindar formación a los docentes en educación para la migración en comunidades de alto índice de expulsión, se puede convertir en una herramienta para generar inclusión social, a través de la difusión de los valores culturales y otras herramientas que permitan a los menores de edad tener diversas perspectivas respecto a su orientación vocacional, no solo la de convertirse en migrante para tener reconocimiento a nivel social en su comunidad.

Los datos encontrados en relación con los aspectos que los sujetos apreciaban sobre su comunidad, apuntarían a la necesidad de fomentar los planteamientos realizados por Toledo y Ortiz-Espejel (2014), quienes tienen registro de que, actualmente, hay más de 2000 proyectos de desarrollo local comunitario que acontecen en el país, y que algunos fungen como posibilidades reales de revertir la migración y repatriar a los migrantes, pues garantizan ingresos económicos para los mismos. Con una perspectiva que no solo parta de las lógicas de producción del modelo neoliberal, se podrían revertir las tendencias a la desertificación del capital social de sus comunidades.

En este sentido, Ceriani y sus colaboradores (2015) también sostienen que es necesario que el Estado mexicano diseñe los planes nacionales de desarrollo con base en el fenómeno de la migración y los derechos humanos de los niños, niñas y adolescentes, incluyendo un incremento del acceso de la educación, para brindar mayor capacitación y formación profesional orientada a responder al mercado laboral en México. 
Por último, si bien la literatura sobre el fenómeno de la migración entre México y EU es amplia, el tema de la relación entre la migración y la educación de los niños, niñas y adolescentes en México aún es un campo con oportunidades de investigar y profundizar para estar en condiciones de proponer políticas públicas y programas pedagógicos. Igualmente, son escasos los estudios respecto a los efectos y consecuencias de los programas y políticas públicas que instituye el Estado. Con base en datos de la ONU (2014), se sostiene que aunque se tienen fuentes de información con datos pertinentes en diversas instituciones nacionales, por lo general esa valiosa información no se utiliza como recurso para la elaboración de las políticas nacionales, por lo que aspirar a "migratizar las políticas de desarrollo", como se señala en el texto coordinado por Arnaut y Giorguli (2010), podría fortalecer y dar una misma dirección a la infraestructura y prácticas de la educación y el desarrollo de empleos en México.

Resulta necesario que los programas que se instituyan con propósitos de fomentar la educación ciudadana en los jóvenes, que contemplen como tema prioritario el problema de la migración con mayor énfasis en las comunidades de expulsión, como es el caso de la comunidad estudiada en este trabajo.

Consideramos que esta aportación consistiría en proporcionar elementos fácticos acerca de cuáles son las conceptualizaciones de los niños y adolescentes sobre la migración para utilizar este conocimiento al momento de diseñar y evaluar programas de educación formal o no formal que consideren lo que el alumno ya conoce, y lo que desconoce, y estar en condiciones de analizar bien la información que le sería necesaria para tomar decisiones tendientes al éxito y al bienestar en cuanto a permanecer en sus comunidades o emigrar buscando mejores oportunidades de vida.

\section{Referencias bibliográficas}

ACNUR. Niños en fuga, México, Alto Comisionado de las Naciones Unidas para los Refugiados, 2013.

Arnaut, A. y Giorguli, S. (Coords.). Los grandes problemas de México. "VII. Educación", México, El Colegio de México, 2010.

Baronnet, B., Mora, M., y Stahler-Sholk, R. (Coords.). Luchas "muy otras" Zapatismo y autonomía en las comunidades in- 
digenas de Chiapas, México, Universidad Autónoma Metropolitana, Xochimilco, 2011.

Barrett, M. y Buchanan-Barrow, E. Children's Understanding of Society, UK Hove, Psychology Press, 2005.

Cárdenas, G. y González, S. Migración no acompañada de niñas, niños y adolescentes. Recuento de una experiencia en México, México, Caminos Posibles, Investigación, Capacitación y Desarrollo S. C., 2013.

Castañeda, E. y Buck, L. "Remittances, Transnational Parenting, and the Children Left Behind: Economic and Psychological Implications", en The Latin Americanist, 55, (4), 85-110, 2011. Disponible en http://doi.org/10.1111/j.1557-203X.2011.01136.x

Ceriani, P. Niñez detenida. Los derechos de los niños, niñas y adolescentes migrantes en la frontera México-Guatemala: diagnóstico y propuestas para pasar del control migratorio a la protección integral de la niñez, México, Distribuciones Fontamara y Universidad Nacional de Lanús, Centro de Derechos Humanos Fray Matías de Córdova, 2013.

Ceriani, P., Musalo, K., y Frydman, L. Niñez y migración en Centro y Norte América: causas, politicas, prácticas y desafíos, Bs. As., Argentina, University of California Hastings College of Law, Programa Migración y Asilo Centro de Justicia y Derechos Humanos Universidad Nacional de Lanús, Argentina, 2015.

$\mathrm{CIDH}$. Derechos humanos de los migrantes y otras personas en el contexto de la movilidad humana en México, México, CIDH, 2013.

CIDH. Situación de los derechos humanos en Mexico. Informe País, México, 2015.

CONAPO. Anuario de Migración y Remesas, México, 2014, México, CONAPO-Fundación BBVA, 2015. Disponible en https://www.bbvaresearch.com/publicaciones/anuariode-migracion-y-remesas-mexico-2015/

Delval, J. El descubrimiento del mundo económico por niños y adolescentes, Madrid, Morata, 2013.

Diez-Martínez, E. "Alfabetización socioeconómica y financiera en adolescentes mexicanos del siglo XXI", en Revista Electrónica de Investigación Educativa, 18 (2), 130-143, 2016. Disponible en http://redie.uabc.mx/redie/article/view/861

Diez-Martinez, E. "Children's understanding of commercial street ads and signs related to economic interchanges", 
en Madruga, A., Enesco, I., Del Barrio, C., y Cohen, R., (Comps.), Construyendo Mentes. Ensayos en homenaje a Juan Delval.Constructing Minds. Essays in tribute to Juan Delval, UNED, Madrid España, 2012.

Diez-Martínez, E., y Ochoa, A. "Análisis de la primera y segunda aspiración ocupacional en la adolescencia: reflexiones sobre la importancia del papel de la escuela y la orientación educativa", en Interacçôes, 9 (26), 2014. Disponible en http://revistas.rcaap.pt/interaccoes/article/view/3368

Diez-Martínez, E., Ochoa, A., y Virués, R. "El desarrollo de las aspiraciones ocupacionales como modelo de estudio sobre la comprensión de la organización social en niños y adolescentes: algunas reflexiones desde la psicología y la educación”, en Revista Latinoamericana de Estudios Educativos, XXXVIII, (1-2), 107-138, México, CEE, 2008.

Domínguez, A. y Polo, A. "Migrantes Potenciales Mexicanos: sus Motivaciones y Actitudes hacia la Aculturación", en Psicología Iberoamericana, 14 (2), 57-62, 2006.

Donato, K. y Duncan, E. "Migration, Social Networks, and Child Health in Mexican Families", en Journal of $\mathrm{Ma}$ rriage and Family, 73 (4), 713-728, 2011. Disponible en http://doi.org/10.1111/j.1741-3737.2011.00841.x

Dreby, J. y Stutz, L. "Making something of the sacrifice: gender, migration and Mexican children's educational aspirations", en Global Networks, 12 (1), 71-90, 2012. http:// doi.org/10.1111/j.1471-0374.2011.00337.x

Durand, J. y Massey, D. Clandestinos Migración México-Estados Unidos en los albores del siglo XXI, México, Miguel Ángel Porrúa, UAZ, col. América Latina y el Nuevo Orden Mundial, 2003.

Enesco, I., Delval, J., y Navarro, A. La comprensión de la organización social en niños y adolescentes, Madrid, Ministerio de Educación y Ciencia 1995.

Furnham, A. y Stacey, B. Young people's understanding of society (vol. XI), Florence, KY, US, Taylor y Frances/Routledge, 1991.

Giorguli, S., Jensen, B., Bean, F., Brown, S., Sawyer, A. y Zuñiga, V. Educational Well-being for Children of Mexican Immigrants in US and in Mexico, México, Brigham Young University y Colegio de México, 2012. 
Giorguli, S. y P. Leite (Coords.). El estado de la migración. Las politicas públicas ante los retos de la migración mexicana a Estados Unidos, Mexico, CONAPO, 2009.

IDC. Dignidad sin Excepción. International Detention Coalition, 2013. Disponible en http://idcoalition.org/es/publications/dignidad-sin-excepcion/

INEGI. Censo de población y vivienda, 2010, México, INEGI, 2010. Disponible en http://www.inegi.org.mx/

Kandel, W. y Kao, G. "The Impact of Temporary Labor Migration on Mexican Children's Educational Aspirations and Performance", en International Migration Review, 35(4),12051231, 2001. http://doi.org/10.1111/j.1747-7379.2001.tb00058.x

Levine, E. “¿Qué pasará con los hijos de los migrantes mexicanos en Estados Unidos", en Veredas, 15, 2007, pp.175-195.

Lu, Y. "Parental Migration and Education of Left-Behind Children: A Comparison of Two Settings", en Journal of Marriage and Family, 76 (5), 1082-1098, 2014. Disponible en http://doi.org/10.1111/jomf.12139

Macías, S. y Reyes, A. "Migración Laboral y Deserción Educativa", en Canadian Journal of Latin American and Caribbean Studies/Revue canadienne des études latino-américaines et caraïbes, 173-202, 2004.

Massey, D., Durand, J., y Malone, N. Detrás de la trama. Políticas migratorias entre México y Estados Unidos, México, Miguel Ángel Porrúa, UAZ, Cámara de Diputados, LIX Legislatura, col. América Latina y el Nuevo Orden Mundial, 2009.

Massey, D., Pren, K., y Durand, J. "Nuevos escenarios de la migración México-Estados Unidos. Las consecuencias de la guerra antiinmigrante", en Papeles Poblac., 15 (61),101-128, 2009.

McKenzie, D., y Rapoport, H. Can Migration Reduce Educational Attainment? Evidence From Mexico, The World Bank, 2006. Disponible en http://elibrary.worldbank.org/doi/ book/10.1596/1813-9450-3952

Muñiz, P. "La situación escolar de niñas y niños rurales en México", en Estudios Demográficos y Urbanos (46), enero-abril, México, El Colegio de México, pp. 53-83, 2001. Disponible en http://www.redalyc.org/articulo.oa?id=31204603 
Ochoa, A. y Diez-Martínez, E. "Relación entre Primera y Segunda Aspiración Ocupacional: ¿Vocación o Educación?”, en Revista Mexicana de Orientación Educativa, 8 (21), 51-59, 2011.

OIM. Informe sobre las migraciones en el mundo, 2013. El bienestar de los migrantes y el desarrollo, Ginebra, Suiza, OIM, 2013. ONU. La situación demográfica en el mundo, Informe conciso, NY, 2014. Pedone, C. Tú siempre jalas a los tuyos. Las cadenas y las redes migratorias de las familias ecuatorianas hacia España, Universitat Autònoma de Barcelona, 2004. Disponible en https://dialnet.unirioja.es/servlet/tesis?codigo=4966\&info $=$ resumen\&idioma $=$ SPA

Portes, A. "Migración y desarrollo: una revisión conceptual de la evidencia", en Migración y desarrollo: perspectivas desde el sur (21-50), 2007.

Questa, A. y Utrilla, B. Otomies del norte del Estado de México y sur de Querétaro, México, CDI, 2006.

Rojas. "Resultados de una política orientada hacia la equidad y calidad de la educación primaria para las niñas y niños jornaleros migrantes”, en Estudios Sociales, 14 (27), 2005.

SEP. Plan de Estudios 2011, Mexico, SEP, 2011.

SEP. Plan Sectorial de Educación 2013-2018, Mexico, SEP, 2013.

SNDIF. Estrategia de Prevención a Niñas, Niños, y Adolescentes Migrantes y Repatriados No acompañados, México, DIF, 2012.

Toledo, V., y Ortiz-Espejel, B. México, regiones que caminan hacia la sustentabilidad. Una Geopolítica de las resistencias bioculturales, México, UIA, Puebla, 2014.

Van Dijk, Sylvia. Las voces de niños, niñas y adolescentes sobre el impacto del proceso migratorio, Reporte de investigación, México, CONCyTEG, 2005.

Vargas, A. y Diez-Martínez, E. (2008). "La comprensión de la organización social en niños y adolescentes: el caso de la migración a los Estados Unidos”, en 20 Congreso Internacional de Migraciones Globales. Experiencias Regionales y esnseñanzas para México, Mazatlán, Sinaloa, México, 24-26 abril de 2008. Disponible en http://interpol.uasnet.mex.mx/migracionesglobales/ 